\title{
Fully Homomorphic Encryption over the Integers
}

\author{
Marten van Dijk ${ }^{1}$, Craig Gentry ${ }^{2}$, Shai Halevi², and Vinod Vaikuntanathan ${ }^{2}$ \\ 1 MIT CSAIL \\ ${ }^{2}$ IBM Research
}

\begin{abstract}
We construct a simple fully homomorphic encryption scheme, using only elementary modular arithmetic. We use Gentry's technique to construct a fully homomorphic scheme from a "bootstrappable" somewhat homomorphic scheme. However, instead of using ideal lattices over a polynomial ring, our bootstrappable encryption scheme merely uses addition and multiplication over the integers. The main appeal of our scheme is the conceptual simplicity.

We reduce the security of our scheme to finding an approximate integer gcd - i.e., given a list of integers that are near-multiples of a hidden integer, output that hidden integer. We investigate the hardness of this task, building on earlier work of Howgrave-Graham.
\end{abstract}

\section{Introduction}

What is the simplest encryption scheme for which one can hope to achieve security? The Caesar cipher is simple, but not secure. We believe that conventional public-key encryption schemes with modular exponentiations are secure, but modular exponentiation is not a very simple operation. If we were to forget our current schemes and start from scratch, perhaps something like the following scheme would be a good candidate for a simple symmetric encryption scheme:

KeyGen: The key is an odd integer, chosen from some interval $p \in\left[2^{\eta-1}, 2^{\eta}\right)$. $\operatorname{Encrypt}(p, m)$ : To encrypt a bit $m \in\{0,1\}$, set the ciphertext as an integer whose residue $\bmod p$ has the same parity as the plaintext. Namely, set $c=$ $p q+2 r+m$, where the integers $q, r$ are chosen at random in some other prescribed intervals, such that $2 r$ is smaller than $p / 2$ in absolute value.

$\operatorname{Decrypt}(p, c):$ Output $(c \bmod p) \bmod 2$.

It is easy to see that when the noise $r$ is sufficiently smaller than the secret key $p$, this simple scheme is both additively and multiplicatively homomorphic for shallow arithmetic circuits. Moreover, one can use Gentry's techniques [6] (i.e., "bootstrapping" and "squashing the decryption circuit") to morph this scheme into a fully homomorphic encryption scheme [20]. Amazingly, it seems that with judicious choice of parameters ( scheme may even be secure!! 
So far we only described a symmetric scheme, but turning it into a public key scheme is easy: The public key consists of many "encryptions of zero", namely integers $x_{i}=q_{i} \cdot p+2 r_{i}$ where $q_{i}, r_{i}$ are chosen from the same prescribed intervals as above. Then to encrypt a bit $m$, the ciphertext is essentially set as $m$ plus a subset sum of the $x_{i}$ 's.

We reduce the security of this scheme to approximate integer gcd - roughly, that it is hard to recover $p$ from the $x_{i}$ 's. This problem, for the case of two $x_{i}$ 's, was analyzed by Howgrave-Graham [9. Our parameters - in particular, the large size of the $q_{i}$ 's - are designed to avoid a generalized version of his attack (as well as other attack avenues, such as solving the associated simultaneous Diophantine approximation problem).

We comment that our scheme is similar to Regev's first encryption scheme [19. In fact, a slight variation of Regev's scheme can be described by exactly the same formula as ours, $\operatorname{Enc}(m, p)=q p+2 r+m$. The main difference between the schemes is that in order to get the homomorphic properties, our choice of parameters is much more aggressive than his. Another difference is that the secret key $p$ in our scheme is an integer, whereas in Regev's scheme the secret key is chosen as an integral fraction of the domain size (i.e., $p=N / h$ for some integer $h$ ). Unfortunately, Regev's worst-to-average-case security reductions from [19] do not seem to apply to our scheme.

\section{Preliminaries}

Below we usually denote parameters by Greek letters (e.g., $\eta, \gamma, \tau$, etc.), with $\lambda$ always denoting the security parameter. Real numbers and integers are denoted by lowercase English letters $(p, q, x, y$, etc.). All logarithms in the text are base-2 unless stated otherwise.

For a real number $z$, we denote by $\lceil z\rceil,\lfloor z\rfloor,\lfloor z\rceil$ the rounding of $a$ up, down, or to the nearest integer. Namely, these are the unique integers in the half open intervals $[z, z+1),(z-1, z]$, and $\left(z-\frac{1}{2}, z+\frac{1}{2}\right]$, respectively.

For a real number $z$ and an integer $p$, we use $q_{p}(z)$ and $r_{p}(z)$ to denote the quotient and remainder of $z$ with respect to $p$, namely $q_{p}(z) \stackrel{\text { def }}{=}\lfloor z / p\rceil$ and $r_{p}(z) \stackrel{\text { def }}{=} z-q_{p}(z) \cdot p$. (Note that $r_{p}(z) \in(-p / 2, p / 2]$.) We also denote the remainder by $[z]_{p}$ or $(z \bmod p)$, we use these three notations interchangeably throughout the paper.

A family $\mathcal{H}$ of hash functions from $X$ to $Y$, both finite sets, is said to be 2-universal if for all distinct $x, x^{\prime} \in X, \operatorname{Pr}_{h}{ }_{\mathfrak{R}}\left[h(x)=h\left(x^{\prime}\right)\right]=1 /|Y|$. A distribution $D$ is $\epsilon$-uniform if its statistical distance from the uniform distribution is at most $\epsilon$, where the statistical difference between two distributions $D_{1}, D_{2}$ over a finite domain $X$ is $\frac{1}{2} \sum_{x \in X}\left|D_{1}(x)-D_{2}(x)\right|$.

Lemma 1 (Simplified Leftover Hash Lemma [8]). Let $\mathcal{H}$ be a family of 2-universal hash functions from $X$ to $Y$. Suppose that $h \stackrel{\mathrm{R}}{\leftarrow} \mathcal{H}$ and $x \stackrel{\mathrm{R}}{\leftarrow} X$ are chosen uniformly and independently. Then, $(h, h(x))$ is $\frac{1}{2} \sqrt{|Y| /|X|}$-uniform over $\mathcal{H} \times Y$. 


\subsection{Homomorphic Encryption}

Our definitions are adapted from Gentry [6]. Below we only consider encryption schemes that are homomorphic with respect to boolean circuits consisting of gates for addition and multiplication mod 2. (Considering only bit operations also means that the plaintext space of the encryption schemes that we consider is limited to $\{0,1\}$.) See the works of Ishai and Paskin [10] for a more general definitional treatment of homomorphic encryption with respect to other forms of "programs."

A homomorphic public key encryption scheme $\mathcal{E}$ has four algorithms: the usual KeyGen, Encrypt, and Decrypt, and an additional algorithm Evaluate. The algorithm Evaluate takes as input a public key pk, a circuit $C$, a tuple of ciphertexts $\boldsymbol{c}=\left\langle c_{1}, \ldots, c_{t}\right\rangle$ (one for every input bit of $C$ ), and outputs another ciphertext $c$.

Definition 1 (Correct Homomorphic Decryption). The scheme $\mathcal{E}=$ (KeyGen, Encrypt, Decrypt, Evaluate) is correct for a given $t$-input circuit $C$ if, for any key-pair (sk, pk) output by KeyGen $(\lambda)$, any t plaintext bits $m_{1}, \ldots, m_{t}$, and any ciphertexts $\boldsymbol{c}=\left\langle c_{1}, \ldots, c_{t}\right\rangle$ with $c_{i} \leftarrow \operatorname{Encrypt}_{\mathcal{E}}\left(\mathrm{pk}, m_{i}\right)$, it is the case that:

$$
\text { Decrypt (sk, Evaluate }(\mathrm{pk}, C, \boldsymbol{c}))=C\left(m_{1}, \ldots, m_{t}\right)
$$

Definition 2 (Homomorphic Encryption). The scheme $\mathcal{E}=($ KeyGen, Encrypt, Decrypt, Evaluate) is homomorphic for a class $\mathcal{C}$ of circuit 1 if it is correct for all circuits $C \in \mathcal{C} . \mathcal{E}$ is fully homomorphic if it is correct for all boolean circuits.

The semantic security of a homomorphic encryption scheme is defined in the usual way [7, without reference to the Evaluate algorithm. (Indeed Evaluate is a public algorithm with no secrets.)

It is clear that as defined above, fully homomorphic encryption can be trivially realized from any secure encryption scheme, by an algorithm Evaluate that simply attaches a description of the circuit $C$ to the ciphertext tuple, and a Decrypt procedure that first decrypts all the ciphertexts and then evaluates $C$ on the corresponding plaintext bits. Two properties of homomorphic encryption that rule out this trivial solution are circuit-privacy and compactness.

Circuit privacy roughly means that the ciphertext generated by Evaluate does not reveal anything about the circuit that it evaluates beyond the output value of that circuit, even for someone who knows the secret key. We discuss circuit privacy in the full version [21. It is folklore that circuit-private fully-homomorphic encryption can be realized using Yao's "garbled circuits" 22 15 and a two-flow oblivious transfer protocol. (This construction is similar to the trivial solution from above, essentially it replaces the plaintext circuit with a garbled circuit.) Hence the "real challenge" in constructing fully homomorphic encryption comes from the compactness property, which essentially means that the size of the ciphertext that Evaluate generates does not depend on the size of the circuit $C$.

Definition 3 (Compact Homomorphic Encryption). The scheme $\mathcal{E}=$ (KeyGen, Encrypt, Decrypt, Evaluate) is compact if there exists a fixed polynomial

\footnotetext{
${ }^{1}$ Formally, $\mathcal{C}$ is an ensemble, parametrized by the security parameter.
} 
bound $b(\lambda)$ so that for any key-pair (sk, pk) output by $\operatorname{KeyGen}(\lambda)$, any circuit $C$ and any sequence of ciphertext $\boldsymbol{c}=\left\langle c_{1}, \ldots, c_{t}\right\rangle$ that was generated with respect to $\mathrm{pk}$, the size of the ciphertext Evaluate $(\mathrm{pk}, C, \boldsymbol{c})$ is not more than $b(\lambda)$ bits (independently of the size of $C$ ).

\subsection{Bootstrappable Encryption}

Following Gentry [6], we construct homomorphic encryption for circuits of any depth from one that is capable of evaluating just a little more than its own decryption circuit.

Definition 4 (Augmented Decryption Circuits). Let $\mathcal{E}=($ KeyGen, Encrypt, Decrypt, Evaluate) be an encryption scheme, where decryption is implemented by a circuit that depends only on the security parameter 2

For a given value of the security parameter $\lambda$, the set of augmented decryption circuits consists of two circuits, both take as input a secret key and two ciphertexts: One circuit decrypts both ciphertexts and adds the resulting plaintext bits mod 2, the other decrypts both ciphertexts and multiplies the resulting plaintext bits $\bmod 2$. We denote this set by $D_{\mathcal{E}}(\lambda)$.

Definition 5 (Bootstrappable Encryption). Let $\mathcal{E}=$ (KeyGen, Encrypt, Decrypt, Evaluate) be a homomorphic encryption scheme, and for every value of the security parameter $\lambda$ let $\mathcal{C}_{\mathcal{E}}(\lambda)$ be a set of circuits with respect to which $\mathcal{E}$ is correct. We say that $\mathcal{E}$ is bootstrappable if $D_{\mathcal{E}}(\lambda) \subseteq \mathcal{C}_{\mathcal{E}}(\lambda)$ holds for every $\lambda$.

Theorem 1 ([6]). There is an (efficient, explicit) transformation that given a description of a bootstrappable scheme $\mathcal{E}$ and a parameter $d=d(\lambda)$, outputs a description of another encryption scheme $\mathcal{E}^{(d)}$ such that:

1. $\mathcal{E}^{(d)}$ is compact (in particular the Decrypt circuit in $\mathcal{E}^{(d)}$ is identical to that in $\mathcal{E})$, and

2. $\mathcal{E}^{(d)}$ is homomorphic for all circuits of depth up to $d$.

Moreover, $\mathcal{E}^{(d)}$ is semantically secure if $\mathcal{E}$ is: Any attack with advantage $\varepsilon$ against $\mathcal{E}^{(d)}$ can be converted into an attack with similar complexity against $\mathcal{E}$ with advantage at least $\varepsilon / \ell d$, where $\ell$ is the length of the secret key in $\mathcal{E}$.

We also note that if the bootstrappable scheme $\mathcal{E}$ is "circular secure" then it can be converted into a single compact fully-homomorphic encryption scheme $\mathcal{E}^{\prime}$. See [6] for details.

\section{A Somewhat Homomorphic Encryption Scheme}

Parameters. The construction below has many parameters, controlling the number of integers in the public key and the bit-length of the various integers.

\footnotetext{
${ }^{2}$ This in particular means that for a fixed value of the security parameter, the size of the secret key is always the same, and similarly all the ciphertexts that can be decrypted have the same size.
} 
Specifically, we use the following four parameters (all polynomial in the security parameter $\lambda)$ :

$\gamma$ is the bit-length of the integers in the public key,

$\eta$ is the bit-length of the secret key (which is the hidden approximate-gcd of all the public-key integers),

$\rho$ is the bit-length of the noise (i.e., the distance between the public key elements and the nearest multiples of the secret key), and

$\tau$ is the number of integers in the public key.

These parameters must be set under the following constraints:

$-\rho=\omega(\log \lambda)$, to protect against brute-force attacks on the noise;

$-\eta \geq \rho \cdot \Theta\left(\lambda \log ^{2} \lambda\right)$, in order to support homomorphism for deep enough circuits to evaluate the "squashed decryption circuit" (cf. Sections 3.2 and 6.2);

$-\gamma=\omega\left(\eta^{2} \log \lambda\right)$, to thwart various lattice-based attacks on the underlying approximate-gcd problem (cf. Section [5);

$-\tau \geq \gamma+\omega(\log \lambda)$, in order to use the leftover hash lemma in the reduction to approximate gcd.

We also use a secondary noise parameter $\rho^{\prime}=\rho+\omega(\log \lambda)$. A convenient parameter set to keep in mind is $\rho=\lambda, \rho^{\prime}=2 \lambda, \eta=\tilde{O}\left(\lambda^{2}\right), \gamma=\tilde{O}\left(\lambda^{5}\right)$ and $\tau=\gamma+\lambda$. (This setting results in a scheme with complexity $\tilde{O}\left(\lambda^{10}\right)$.)

For a specific $(\eta$-bit) odd positive integer $p$, we use the following distribution over $\gamma$-bit integers:

$\mathcal{D}_{\gamma, \rho}(p)=\left\{\right.$ choose $q \stackrel{\$}{\longleftarrow} \mathbb{Z} \cap\left[0,2^{\gamma} / p\right), r \stackrel{\$}{\longleftarrow} \mathbb{Z} \cap\left(-2^{\rho}, 2^{\rho}\right)$ : output $\left.x=p q+r\right\}$

This distribution is clearly efficiently sampleable.

\subsection{The Construction}

KeyGen $(\lambda)$. The secret key is an odd $\eta$-bit integer: $p \stackrel{\$}{\leftarrow}(2 \mathbb{Z}+1) \cap\left[2^{\eta-1}, 2^{\eta}\right)$.

For the public key, sample $x_{i} \stackrel{\$}{\leftarrow} \mathcal{D}_{\gamma, \rho}(p)$ for $i=0, \ldots, \tau$. Relabel so that $x_{0}$ is the largest. Restart unless $x_{0}$ is odd and $r_{p}\left(x_{0}\right)$ is even. The public key is $\mathrm{pk}=\left\langle x_{0}, x_{1}, \ldots, x_{\tau}\right\rangle$.

Encrypt (pk, $m \in\{0,1\})$. Choose a random subset $S \subseteq\{1,2, \ldots, \tau\}$ and a random integer $r$ in $\left(-2^{\rho^{\prime}}, 2^{\rho^{\prime}}\right)$, and output $c \leftarrow\left[m+2 r+2 \sum_{i \in S} x_{i}\right]_{x_{0}}$.

Evaluate $\left(\mathbf{p k}, C, c_{1}, \ldots, c_{t}\right)$. Given the (binary) circuit $\mathcal{C}_{\mathcal{E}}$ with $t$ inputs, and $t$ ciphertexts $c_{i}$, apply the (integer) addition and multiplication gates of $\mathcal{C}_{\mathcal{E}}$ to the ciphertexts, performing all the operations over the integers, and return the resulting integer.

Decrypt $(\mathbf{s k}, c)$. Output $m^{\prime} \leftarrow(c \bmod p) \bmod 2$.

Remark 1. Recall that $(c \bmod p)=c-p \cdot\lfloor c / p\rceil$, and as $p$ is odd we can instead decrypt using the formula $m^{\prime} \leftarrow[c-\lfloor c / p\rceil]_{2}=(c \bmod 2) \oplus(\lfloor c / p\rceil \bmod 2)$.

Remark 2. Originally, we described encryption as adding $m$ to a random subset sum of "encryptions of zero". Indeed, the scheme can viewed this way. Let $w_{i}=$ $\left[2 x_{i}\right]_{x_{0}}$ for $i=1, \ldots, \tau$. Each $w_{i}$, and also $x_{0}$, is essentially an encryption of zero; its noise is even. Moreover, $c=m+2 r+\sum_{i \in S} w_{i}-k \cdot x_{0}$ for some integer $k$. 


\subsection{Correctness}

Permitted Circuits and Polynomials. For a mod-2 arithmetic circuit (composed of mod-2 Add and Mult gates), we consider its generalization to the integers, i.e., the same circuits with the Add and Mult gates applied to integers rather than to bits. Similar to Gentry [6], we define a permitted circuit as one where for any $\alpha \geq 1$ and any set of integer inputs all less than $2^{\alpha\left(\rho^{\prime}+2\right)}$ in absolute value, it holds that the generalized circuit's output has absolute value at most $2^{\alpha(\eta-4)}$. Let $\mathcal{C}_{\mathcal{E}}$ denote the set of permitted circuits. Clearly, we have:

Lemma 2. The scheme from above is correct for $\mathcal{C}_{\mathcal{E}}$.

Remark 3. Since "fresh" ciphertexts output by Encrypt have noise at most $2^{\rho^{\prime}+2}$, the ciphertext output by Evaluate applied to a permitted circuit has noise at most $2^{\eta-4}<p / 8$. The bound $2^{\eta-2}<p / 2$ would suffice for correct decryption. But we will later use the fact that the noise remains below $p / 8$ in Section 6 to perform the decryption operation using a very shallow arithmetic circuit.

The definition of the set $\mathcal{C}_{\mathcal{E}}$ from above is rather indirect. In particular this definition does not give a good picture of what $\mathcal{C}_{\mathcal{E}}$ "looks like". By the triangle inequality, a $k$-fan-in Add gate clearly increases the magnitude of the integers by at most a factor of $k$. However, a 2-fan-in Mult gate may square the magnitude of the integers - i.e., double their bit-lengths. So, clearly, the main bottleneck is the multiplicative depth of the circuit, or the degree of the multivariate polynomial computed by the circuit. We have the following lemma.

Lemma 3. Let $C$ be a boolean circuit with $t$ inputs, and let $C^{\dagger}$ be the associated integer circuit (where boolean gates are replaced with integer operations). Let $f\left(x_{1}, \ldots, x_{t}\right)$ be the multivariate polynomial computed by $C^{\dagger}$; let $d$ be its degree. If $|\boldsymbol{f}| \cdot\left(2^{\rho^{\prime}+2}\right)^{d} \leq 2^{\eta-4}$ (where $|\boldsymbol{f}|$ is the $l_{1}$ norm of the coefficient vector of $f$ ) then $C \in \mathcal{C}_{\mathcal{E}}$.

In particular, $\mathcal{E}$ can handle $f$ as long as

$$
d \leq \frac{\eta-4-\log |\boldsymbol{f}|}{\rho^{\prime}+2}
$$

Below we refer to polynomials that satisfy Equation (11) as permitted polynomials and we denote by $\mathcal{P}_{\mathcal{E}}$ the set of permitted polynomials and by $C\left(\mathcal{P}_{\mathcal{E}}\right)$ the set of circuits that compute them. The discussion above implies that $C\left(\mathcal{P}_{\mathcal{E}}\right) \subseteq \mathcal{C}_{\mathcal{E}}$.

Remark 4. For our purposes, we consider settings where $\log |\boldsymbol{f}|$ is small in relation to $\eta, \rho^{\prime}=\omega(\log \lambda)$ and $t, \tau \leq \lambda^{\beta}$, and we need to support polynomials of degree up to $\alpha \lambda \log ^{2} \lambda$ (for some constants $\alpha, \beta$ ). Plugging these expressions in Equation (11), it is sufficient to set $\eta=\rho^{\prime} \cdot \Theta\left(\lambda \log ^{2} \lambda\right)$. 


\subsection{Optimizations}

Modular-reduction during Evaluate. Note that while Encrypt reduces the ciphertext modulo the public key element $x_{0}$, we cannot do the same in Evaluate. The reason is that after just one multiplication the ciphertext becomes much larger than $x_{0}$, so modular reduction will include a large multiple of $x_{0}$ hence introducing intolerable error.

To reduce the ciphertext size during Evaluate, we can add to the public key more elements of the form $x_{i}^{\prime}=q_{i}^{\prime} p+2 r_{i}^{\prime}$ where the $r_{i}^{\prime}$ 's are chosen as usual from the interval $\left(-2^{\rho}, 2^{\rho}\right)$ but the $q_{i}$ 's are chosen much larger than for the other public key elements. Specifically, for $i=0, \ldots \gamma$, we set:

$$
q_{i}^{\prime} \stackrel{\$}{\leftarrow} \cap\left[2^{\gamma+i-1} / p, 2^{\gamma+i} / p\right), \quad r_{i}^{\prime} \stackrel{\$}{\leftarrow} \cap\left(-2^{\rho}, 2^{\rho}\right), \quad x_{i}^{\prime} \leftarrow 2\left(q_{i}^{\prime} \cdot p+r_{i}^{\prime}\right),
$$

thus getting $x_{i}^{\prime} \in\left[2^{\gamma+i}, 2^{\gamma+i+1}\right]$. During Evaluate, every time we have a ciphertext that grows beyond $2^{\gamma}$, we reduce it first modulo $x_{\gamma}^{\prime}$, then modulo $x_{\gamma-1}^{\prime}$, and so on all the way down to $x_{0}^{\prime}$, at which point we again have a ciphertext of bit-length no more than $\gamma$.

Recall that a single operation at most doubles the bit-length of the ciphertext. Hence after any one operation the ciphertext cannot be larger than $2 x_{\gamma}^{\prime}$, and therefore the sequence of modular reductions involves only small multiples of the $x_{i}^{\prime}$ 's, which means that it only adds a small amount of noise. (We note that in addition to smaller ciphertexts, this optimization also reduces the public key size when we use the "decryption squashing" technique as described in Section 6.1.)

It is not clear to what extent adding these larger integers to the public key influences the security of the scheme. It does change the specifics of the approximate-GCD assumption that we need to make, but the same decision-tosearch reduction from Section 4 still goes through 3 Also, we note that having integers with these very large quotients does not seem to help in any of the attacks on approximate-GCD that we considered.

Remark 5. Note that when using the original scheme without the optimization, homomorphic evaluation of different circuits that compute the same polynomial would result in the exact same output ciphertext (i.e., the polynomial applied to the input ciphertexts over the integers). This is no longer true when using the size-reduction optimization, because of the additional modular reduction steps. For example, evaluating the circuit " $x_{1}\left(x_{2}+x_{3}\right)$ " is likely to yield a different ciphertext than the circuit " $x_{1} x_{2}+x_{1} x_{3}$."

In principle, it is plausible that evaluating one circuit would yield a ciphertext with small enough noise to be decrypted, while evaluating another circuit for the same polynomial will produce a ciphertext with too much noise. Adapting the "bootstrappability analysis" from Section [6.2 to the optimized scheme, one would have to take into account not only the degree of the polynomial implementing the decryption process but also the particular circuit that implements this polynomial. It should not be hard to argue that the circuit in Section 6.2 does not introduce too much noise, but the analysis is quite tedious and is omitted here.

${ }^{3}$ Allowing this reduction to go through is the reason that the $x_{i}^{\prime}$ 's are set as even integers. 
Ciphertext compression. Even though the optimization from above keeps evaluated ciphertexts at the same length as original ciphertexts, the size of these ciphertexts is still very large $-\tilde{\theta}\left(\lambda^{5}\right)$ bits under our suggested parameters. We next show how to "compress", or post-process the ciphertexts, down to (asymptotically) the size of an RSA modulus, reducing the communication complexity of our scheme dramatically.

The price of this optimization, however, is that we cannot evaluate anything on these compressed ciphertexts. Hence we can only use this compression technique on the final output ciphertexts, after all applications of the Evaluate algorithm have been completed. (This technique also introduces another hardness assumption, similar to the $\phi$-hiding assumption of Cachin et al. 3].)

Roughly, we supplement the public key with the description of a group $G$ and an element $g \in G$ whose order is a multiple of the secret key $p$. Then, given the ciphertext $c$ from our scheme, the compressed ciphertext is simply $c^{*} \leftarrow g^{c}$. Note that $\mathrm{DL}_{g}\left(c^{*}\right)=c(\bmod p)$, so decrypting is done by first computing $y \leftarrow \mathrm{DL}_{g}\left(c^{*}\right) \bmod p$, and then $m \leftarrow y \bmod 2$. Correctness follows immediately from the correctness of the original scheme.

To implement this idea, we need to choose the secret key $p$ as a smooth number so that we can compute $\left(\mathrm{DL}_{g}\left(c^{*}\right) \bmod p\right)$ on decryption. It seems sufficient to choose the secret key as a product of random distinct $\lambda^{2} / \log \lambda$ small primes (say, all smaller than $\lambda^{3}$ ). Also, we need to ensure that publishing $G, g$ does not violate the security of the scheme. This can be accomplished by publishing an RSA modulus $N$ such that $p \mid \phi(N)$ (and $\log N$ sufficiently larger than $4 \log p$ ) 4 along with a random element $g \in_{R} Z_{N}^{*}$, relying on a variant of the $\phi$-hiding assumption 3]. Namely, we assume that given two smooth numbers $p_{1}, p_{2}$ as above and given $N$ such that one of the $p_{i}$ 's divides $\phi(N)$, it is hard to determine which of the two $p_{i}$ 's divides $\phi(N)$. In the full version we describe this optimization in more details, and provide a proof of security for it under this $\phi$-hiding variant.

\section{Security of the Somewhat Homomorphic Scheme}

We reduce the security of the scheme from Section 3 to the hardness of the approximate-gcd problem. Namely, given a set of integers $x_{0}, x_{1}, \ldots, x_{\tau}$, all randomly chosen close to multiples of a large integer $p$, find this "common near divisor" $p$.

On a high level, our reduction resembles classical hard-core-bit proofs in factoring-based cryptography (e.g., Alexi et al. [1]): Fixing a randomly-chosen public key, we roughly show that an adversary who can predict the encrypted bit in a random ciphertext under this public key can be used to find the secret key (for this fixed public key). As in 11, we describe a random-self-reduction and accuracy-amplification step that uses the promised adversary to get a reliable oracle for the least-significant bit, and then a binary-GCD algorithm that uses that reliable oracle to find $p$.

${ }^{4}$ The condition $\log N>4 \log p$ is needed, since otherwise we can use Coppersmith's method 4] to break the corresponding $\phi$-hiding assumption. 
The technical details, of course, are very different than in factoring-based cryptography. Perhaps the main difference is that our random self-reduction entails a loss in parameters. Specifically, we show that a noticeable advantage in guessing the encrypted bit in a random "high noise ciphertext" - where the noise is $\rho^{\prime}$ bits - can be converted into the ability to predict reliably the parity bit of the quotient in an arbitrary "low noise integer" - where the noise is $\rho$ bits. (Roughly, the reason for this is that we need to add extra noise to "wipe out the traces" of the non-random noise in the arbitrary input integer.)

The implication is that we can only reduce the security of our cryptosystem in the "high-noise regime" to the hardness of approximate-gcd in the "low-noise regime." Note that the difference between "high noise" and "low noise" is rather small: only $\omega(\log \lambda)$ bits.

\subsection{Reduction to Approximate-GCD}

The approximate-gcd problem is defined as follows:

Definition 6 (Approximate GCD). The $(\rho, \eta, \gamma)$-approximate-gcd problem is: given polynomially many samples from $\mathcal{D}_{\gamma, \rho}(p)$ for a randomly chosen $\eta$-bit odd integer $p$, output $p$.

Theorem 2. Fix the parameters $\left(\rho, \rho^{\prime}, \eta, \gamma, \tau\right)$ as in the Somewhat Homomorphic Scheme from Section 3 (all polynomial in the security parameter $\lambda$ ).

Any attack $\mathcal{A}$ with advantage $\varepsilon$ on the encryption scheme can be converted into an algorithm $\mathcal{B}$ for solving $(\rho, \eta, \gamma)$-approximate-gcd with success probability at least $\varepsilon / 2$. The running time of $\mathcal{B}$ is polynomial in the running time of $\mathcal{A}$, and in $\lambda$ and $1 / \epsilon$.

Proof. Recall that we use $q_{p}(z)$ and $r_{p}(z)$ to denote the quotient and remainder of $z$ with respect to $p$, hence $z=q_{p}(z) \cdot p+r_{p}(z)$. Let $\mathcal{A}$ be an attacker against the scheme. Namely, $\mathcal{A}$ takes as input a public key and a ciphertext (as produced by KeyGen and Encrypt of our scheme), and outputs the correct plaintext bit with probability $\frac{1}{2}+\epsilon$ for some noticeable $\epsilon$. (The probability is over KeyGen and Encrypt, as well as the choice of the plaintext bit and the internal randomness of $\mathcal{A}$.)

We use $\mathcal{A}$ to construct a solver $\mathcal{B}$ for approximate-gcd with parameters $\rho, \eta, \gamma$. For a randomly chosen $\eta$-bit odd integer $p$, the solver $\mathcal{B}$ has access to as many samples from $\mathcal{D}_{\gamma, \rho}(p)$ as it needs, and the goal is to find $p$.

Step 1: Creating a public key. The solver $\mathcal{B}$ begins by constructing a public key for the scheme. $\mathcal{B}$ draws $\tau+1$ samples $x_{0}, \ldots, x_{\tau} \stackrel{\$}{\leftarrow} \mathcal{D}_{\gamma, \rho}(p)$. It relabels so that $x_{0}$ is the largest. It restarts unless $x_{0}$ is odd. $\mathcal{B}$ then outputs a public key $\mathrm{pk}=\left\langle x_{0}, x_{1}, \ldots, x_{\tau}\right\rangle$. Clearly, if $r_{p}\left(x_{0}\right)$ happens to be even then the distribution induced on the public key is identical to that of the scheme.

Step 2: A subroutine for high-accuracy LSB predictor. Next, $\mathcal{B}$ produces a sequence of integers, and attempts to recover $p$ by utilizing $\mathcal{A}$ to learn the leastsignificant bit of the quotients of these integers with respect to $p$. For this, $\mathcal{B}$ uses the following subroutine: 
Subroutine Learn-LSB $(z, \mathrm{pk})$ :

$\overline{\text { Input: } z \in\left[0,2^{\gamma}\right) \text { with }\left|r_{p}(z)\right|}<2^{\rho}$, a public key pk $=\left\langle x_{0}, x_{1}, \ldots, x_{\tau}\right\rangle$

Output: The least-significant-bit of $q_{p}(z)$

1. For $j=1$ to $\operatorname{poly}(\lambda) / \epsilon$ do:

$/ / \epsilon$ is the overall advantage of $\mathcal{A}$

2. Choose noise $r_{j} \stackrel{\$}{\leftarrow}\left(-2^{\rho^{\prime}}, 2^{\rho^{\prime}}\right)$, a bit $m_{j} \stackrel{\$}{\leftarrow}\{0,1\}$,

3. Set $c_{j} \leftarrow\left[z+m_{j}+2 r_{j}+2 \sum_{k \in S_{j}} x_{k}\right]_{x_{0}}$

4. $\quad$ Call $\mathcal{A}$ to get a prediction $a_{j} \leftarrow \mathcal{A}\left(\mathrm{pk}, c_{j}\right)$

5. Set $b_{j} \leftarrow a_{j} \oplus \operatorname{parity}(z) \oplus m_{j} \quad / / b_{j}$ should be the parity of $q_{p}(z)$

6. Output the majority vote among the $b_{j}$ 's.

In the full version [21] we show that for all but a negligible fraction of the public keys generated by the scheme, the "ciphertext" $c_{j}$ in line 3 is distributed almost identically to a valid encryption of the bit $\left[r_{p}(z)\right]_{2} \oplus m_{j}$. Note also that since $p$ is odd, we always have $\left[q_{p}(z)\right]_{2}=\left[r_{p}(z)\right]_{2} \oplus$ parity $(z)$. It follows that if $\mathcal{A}$ has a noticeable advantage in guessing the encrypted bit under pk then $\operatorname{Learn}-\operatorname{LSB}(z, \mathrm{pk})$ will return $\left[q_{p}(z)\right]_{2}$ with overwhelming probability.

Step 3: Binary $G C D$. Once we turned $\mathcal{A}$ into an oracle for the least-significantbit of $q_{p}(z)$, recovering $p$ is rather straightforward. Perhaps the simplest way of doing it is using the binary GCD algorithm [12]: Given any two integers $z_{1}=q_{p}\left(z_{1}\right) \cdot p+r_{p}\left(z_{1}\right)$ and $z_{2}=q_{p}\left(z_{2}\right) \cdot p+r_{p}\left(z_{2}\right)$ (with $r_{p}\left(z_{i}\right) \ll p$ ), repeatedly apply the following process to them:

1. If $z_{2}>z_{1}$ then swap them, $z_{1} \leftrightarrow z_{2}$.

2 . Use the oracle to learn the parity bit of both $q_{p}\left(z_{1}\right)$ and $q_{p}\left(z_{2}\right)$, denote $b_{i}=\left[q_{p}\left(z_{i}\right)\right]_{2}$.

3. If both $q_{p}\left(z_{i}\right)$ are odd then replace $z_{1}$ by $z_{1} \leftarrow z_{1}-z_{2}$ and set $b_{1} \leftarrow 0$.

4. For each $z_{i}$ with $b_{i}=0$, replace $z_{i}$ by $z_{i} \leftarrow\left(z_{i}-\operatorname{parity}\left(z_{i}\right)\right) / 2$. (Note that $z_{i}-\operatorname{parity}\left(z_{i}\right)$ is even, so the new $z_{i}$ is an integer.)

Observe that when $p \gg r_{p}\left(z_{i}\right)$, subtracting the parity bit does not change the quotient with respect to $p$, only the remainder. That is, $q_{p}\left(z_{i}-\operatorname{parity}\left(z_{i}\right)\right)=$ $q_{p}\left(z_{i}\right)$. It follows that when we set $z_{i}^{\prime} \leftarrow\left(z_{i}-\operatorname{parity}\left(z_{i}\right)\right) / 2$ in line 4 (where we know that $q_{p}\left(z_{i}\right)$ is even), we get

$$
q_{p}\left(z_{i}^{\prime}\right)=q_{p}\left(z_{i}\right) / 2 \text { and } r_{p}\left(z_{i}^{\prime}\right)=\left(r_{p}\left(z_{i}\right)-\operatorname{parity}\left(z_{i}\right)\right) / 2 .
$$

We now show that the noise in $z_{1}, z_{2}$ never grows too large in this process. Clearly, setting $z_{i}^{\prime} \leftarrow\left(z_{i}-\right.$ parity $\left.\left(z_{i}\right)\right) / 2$ in line 4 we have $\left|r_{p}\left(z_{i}^{\prime}\right)\right| \leq\left(\left|r_{p}\left(z_{i}\right)\right|+1\right) / 2 \leq$ $\left|r_{p}\left(z_{i}\right)\right|$. Moreover, when we replace $z_{1}$ by $z_{1}^{\prime} \leftarrow z_{1}-z_{2}$ in line 3 and then by $z_{1}^{\prime \prime} \leftarrow\left(z_{1}^{\prime}-\operatorname{parity}\left(z_{1}^{\prime}\right)\right) / 2$ in line 4 , we have

$$
\left|r_{p}\left(z_{1}^{\prime \prime}\right)\right|=\left(\left|r_{p}\left(z_{1}\right)-r_{p}\left(z_{2}\right)-\operatorname{parity}\left(z_{1}^{\prime}\right)\right|\right) / 2 \leq \max \left\{\left|r_{p}\left(z_{1}\right)\right|,\left|r_{p}\left(z_{2}\right)\right|\right\}
$$

Hence the $r_{p}\left(z_{i}\right)$ 's never grow beyond the largest of the initial two, so we always have $p \gg r_{p}\left(z_{i}\right)$. 
This implies that the operations above correspond to the usual operations of the binary GCD algorithm, applied to the $q_{p}\left(z_{i}\right)$ 's. Hence after $O(\gamma)$ iterations we will finally get two integers $z_{1}^{\prime}, z_{2}^{\prime}$ with $z_{2}^{\prime}=0$ and $q_{p}\left(z_{1}^{\prime}\right)$ being the odd part of $G C D\left(q_{p}\left(z_{1}\right), q_{p}\left(z_{2}\right)\right)$ (for the two initial integers).

Step 4: Recovering $\mathbf{p}$. To recover $p$, the solver $\mathcal{B}$ draws a pair of elements $z_{1}^{*}, z_{2}^{*} \stackrel{\$}{\longleftarrow}$ $\mathcal{D}_{\gamma, \rho}(p)$ and applies the binary-GCD algorithm to them. With probability at least $\pi^{2} / 6 \approx 0.6$, the odd part of $G C D\left(q_{p}\left(z_{1}^{*}\right), q_{p}\left(z_{2}^{*}\right)\right)$ is one, which means that the procedure will output an element $\tilde{z}=1 \cdot p+r$ with $|r| \leq 2^{\rho}$. (If this does not happen then $\mathcal{B}$ draws two new integers and tries again.)

Lastly $\mathcal{B}$ repeats the binary-GCD procedure from above using $z_{1}=z_{1}^{*}$ and $z_{2}=\tilde{z}$, and the sequence of parity bits of the $q_{p}\left(z_{1}\right)$ 's in all the iterations spell out the binary representation of $q_{p}\left(z_{1}^{*}\right)$. Now $\mathcal{B}$ recovers $p=\left\lfloor z_{1}^{*} / q_{p}\left(z_{1}^{*}\right)\right\rceil$.

Summary. We have shown that $\mathcal{B}$ can recover $p$ given access to a reliable oracle for computing $\left[q_{p}(z)\right]_{2}$ (for $z$ 's with noise much smaller than $p$ ). It is left to analyze the probability (over $\mathcal{B}$ 's choice of public key) with which the procedure Learn- $\operatorname{LSB}(z, \mathrm{pk})$ from above is indeed such a reliable oracle.

The Success Probability of $\mathcal{B}$. In the full version we prove a simple technical lemma about the distribution of ciphertexts in our scheme. Recall that conditioned on some probability- $\frac{1}{2}$ event in our reduction (i.e., $q_{p}\left(x_{0}\right)$ is odd), the distribution of the public key that $\mathcal{B}$ generates is identical to the correct distribution from the scheme. Let us denote this probability- $\frac{1}{2}$ "good event" by $\mathcal{G}$. In the full version we prove that for every secret key $p$ and for all but a negligible fraction of the public keys (as generated by KeyGen for the secret key $p$ ), the procedure that $\mathcal{B}$ uses to generate ciphertexts in line 3 of the subroutine Learn-LSB produces a distribution which is statistically close to the ciphertext distribution of the scheme. This lets us analyze the success probability of $\mathcal{B}$, as follows: Let $\mathcal{P}$ be the set of odd integers in $\left[2^{\eta-1}, 2^{\eta}\right)$ for which $\mathcal{A}$ has more than $\varepsilon / 2$ advantage

$$
\mathcal{P} \stackrel{\text { def }}{=}\left\{p \in\left[2^{\eta-1}, 2^{\eta}\right): \text { advantage }(\mathcal{A}) \text { conditioned on sk }=p \text { is at least } \varepsilon / 2\right\}
$$

A counting argument shows that the fraction of odd integers from $\left[2^{\eta-1}, 2^{\eta}\right)$ that are in $\mathcal{P}$ is at least $\varepsilon / 2$. For a given $p \in \mathcal{P}$, we similarly denote by $\mathcal{P} \mathcal{K}_{p}$ the set of public keys for which $\mathcal{A}$ has advantage at least $\varepsilon / 4$ :

$$
\mathcal{P} \mathcal{K}_{p} \stackrel{\text { def }}{=}\{\mathrm{pk} \text { for } p: \text { advantage }(\mathcal{A}) \text { conditioned on pk is at least } \varepsilon / 4\}
$$

Again, for every $p \in \mathcal{P}$, the KeyGen algorithm (when using the secret key sk $=p$ ) must output pk $\in \mathcal{P} \mathcal{K}_{p}$ with probability at least $\varepsilon / 4$.

Consider now a single run of $\mathcal{B}$ when it is given access to $\mathcal{D}_{\gamma, \rho}(p)$ for some $p \in \mathcal{P}$. With probability $1 / 2$ the "good event" $\mathcal{G}$ happens, in which case the public key that $\mathcal{B}$ produces is negligibly close to the right distribution. Hence conditioned on $\mathcal{G}, \mathcal{B}$ generates some pk $\in \mathcal{P} \mathcal{K}_{p}$ with probability $\varepsilon^{\prime} \geq \varepsilon / 4-$ negl. Moreover, with probability $\varepsilon^{\prime}-$ negl not only is the public key in $\mathcal{P} \mathcal{K}_{p}$, but also the ciphertext-generation that $\mathcal{B}$ uses in line 3 of Learn-LSB "works" for 
this public key (meaning that the ciphertexts that it generates are chosen from almost the right distribution). If that happens then $\mathcal{A}$ returns the right answer in line 4 of Learn-LSB with probability $\varepsilon / 4-$ negl. As that subroutine calls $\mathcal{A}$ for $\operatorname{poly}(\lambda) / \varepsilon$ times and takes majority vote, it will return the right answer with overwhelming probability, and $\mathcal{B}$ will recover the approximate-gcd $p$.

Thus, when the hidden secret is $p \in \mathcal{P}$ then $\mathcal{B}$ has probability at least $1 / 2$. $(\varepsilon / 4-$ negl) of recovering it in a single run. Repeating the algorithm $\mathcal{B}$ for $(8 / \epsilon)$. $\omega(\log \lambda)$ times will therefore recover such $p$ 's with overwhelming probability. Hence we have a solver of complexity $\operatorname{poly}(\lambda, 1 / \varepsilon)$ that works with overwhelming probability for every $p \in \mathcal{P}$, so the overall success probability of this solver is at least the density of $\mathcal{P}$, which is at least $\varepsilon / 2$. This completes the proof of Theorem 2,

\section{Known Attacks}

Consider the approximate-gcd instance $\left\{x_{0}, \ldots, x_{t}\right\}$ where $x_{i}=p q_{i}+r_{i}$. In this section, we first review known attacks on the approximate-gcd problem for two numbers (i.e., when $t=1$ ) - including brute-forcing the remainders, continued fractions, and Howgrave-Graham's approximate gcd algorithm 9]. Later, we consider attacks for arbitrarily large values of $t$ - including lattice-based algorithms for simultaneous Diophantine approximation [13, Nguyen and Stern's orthogonal lattice [17, and extensions of Coppersmith's method to multivariate polynomials [4.

\subsection{The Approximate GCD of Two Numbers}

A simple brute-force attack is to try to guess $r_{1}$ and $r_{2}$, and verify the guess with a gcd computation. Specifically, for $r_{1}^{\prime}, r_{2}^{\prime} \in\left(-2^{\rho}, 2^{\rho}\right)$, set

$$
x_{1}^{\prime} \leftarrow x_{1}-r_{1}^{\prime}, \quad x_{2}^{\prime} \leftarrow x_{2}-r_{2}^{\prime}, \quad p^{\prime} \leftarrow \operatorname{gcd}\left(x_{1}^{\prime}, x_{2}^{\prime}\right)
$$

If $p^{\prime}$ has $\eta$ bits, output $p^{\prime}$ as a possible solution. The solution $p$ will definitely be found by this technique, and for our parameter choices, where $\rho$ is much smaller than $\eta$, the solution is likely to be unique. The running time of the attack is approximately $2^{2 \rho}$.

A variant of the brute-force attack is to set $x_{1}^{\prime}$ as above, factor $x_{1}^{\prime}$, and, if there is an $\eta$-bit factor $p^{\prime}$, see whether $p^{\prime}$ is an approximate divisor of $x_{2}^{\prime}$. Since in our parameters $\gamma$ is substantially greater than $\eta$, the attack should use a factoring algorithm whose performance depends primarily on the size of the target factor rather than the size of the entire number being factored. For example, Lenstra's elliptic curve factoring algorithm [14] runs in time roughly $\exp (O(\sqrt{\eta})$ ) (with only polynomial dependence on $\gamma$ ), thus resulting in overall attack complexity $\approx 2^{\rho+\sqrt{\eta}}$. The attack time is less if the approximate ged is known to be smooth, but still exponential in $\rho$.

Continued fractions seem like a natural way to recover $p$ from $x_{1}$ and $x_{2}$. Using continued fractions, one obtains a sequence of integer pairs $\left(a_{i}, b_{i}\right)$ such that 
$\left|x_{1} / x_{2}-a_{i} / b_{i}\right|<1 / b_{i}^{2}$. Moreover, every pair $(s, t)$ such that $\left|x_{1} / x_{2}-s / t\right|<1 / 2 t^{2}$ is in the sequence. Since $q_{1} / q_{2}$ is a good approximation of $x_{1} / x_{2}$, one may hope that it occurs as a pair in the sequence; if so, one recovers $p=\left\lfloor x_{1} / q_{1}\right\rceil$. However, in our scheme, $\left|x_{1} / x_{2}-q_{1} / q_{2}\right|$ is not small enough to be recovered using continued fractions. Specifically, we have

$$
\left|\frac{x_{1}}{x_{2}}-\frac{q_{1}}{q_{2}}\right|=\left|\frac{q_{2} r_{1}-q_{1} r_{2}}{q_{2}\left(p q_{2}+r_{2}\right)}\right| \approx\left|\frac{q_{2} r_{1}-q_{1} r_{2}}{p}\right| \cdot \frac{1}{q_{2}^{2}}
$$

where $\left(q_{2} r_{1}-q_{1} r_{2}\right) / p$ in the final term is likely to be much larger than 1 . To describe the failure of continued fractions another way, the mere fact that an approximant $a_{i} / b_{i}$ is close to $x_{1} / x_{2}$ does not mean that there exist $r_{1}^{\prime}, r_{2}^{\prime} \ll p$ such that $x_{1}=p a_{i}+r_{1}^{\prime}$ and $x_{2}=p b_{i}+r_{2}^{\prime}$ - i.e., the continued fractions method is not constrained to output the kind of approximants that we need. See [9] for a more detailed exposition of the continued fractions approach to approximate-gcd.

Howgrave-Graham [9] also gives a lattice attack on the two-element approximate-gcd problem that is related to Coppersmith's celebrated algorithm for finding small solutions to univariate and bivariate modular equations [4]. For the case where $x_{1}$ is exactly divisible by $p$, where his algorithm performs slightly better, the attack recovers $p$ when $\rho / \gamma$ is smaller than $(\eta / \gamma)^{2}$. The algorithm does not degrade gracefully for $\rho, \eta, \gamma$ that do not satisfy the constraint. Rather, in this case, the relevant lattice may contain exponentially vectors unrelated to the approximate-gcd solution, so that lattice reduction yields nothing useful.

\subsection{The Approximate GCD of Many Numbers}

Now, let us consider attacks - specifically, lattice attacks - for arbitrary $t$. First, note that the rational numbers $y_{i}=x_{i} / x_{0}$ are an instance of the simultaneous Diophantine approximation (SDA) problem: indeed for all $i$ it holds that $\frac{x_{i}}{x_{0}}=$ $\frac{q_{i}+s_{i}}{q_{0}}$, where $\left|s_{i}\right| \approx 2^{\rho-\eta}$. We can therefore try to use Lagarias' algorithm for SDA [13, namely apply LLL to the $(t+1)$-dimensional lattice $L$ spanned by the rows of the following matrix:

$$
M=\left(\begin{array}{ccccc}
2^{\rho} & x_{1} & x_{2} & \ldots & x_{t} \\
& -x_{0} & & & \\
& & -x_{0} & & \\
& & & \ddots & \\
& & & & -x_{0}
\end{array}\right)
$$

Our target solution corresponds to a vector of length roughly $2^{\gamma+\rho-\eta} \sqrt{t+1}-$ specifically,

$$
\begin{aligned}
\boldsymbol{v}=\left\langle q_{0}, q_{1}, \ldots, q_{t}\right\rangle \cdot M & =\left\langle q_{0} 2^{\rho}, q_{0} x_{1}-q_{1} x_{0}, \ldots, q_{0} x_{t}-q_{t} x_{0}\right\rangle \\
& =\left\langle q_{0} 2^{\rho}, x_{0} q_{0}\left(\frac{x_{1}}{x_{0}}-\frac{q_{1}}{q_{0}}\right), \ldots, x_{0} q_{0}\left(\frac{x_{t}}{x_{0}}-\frac{q_{t}}{q_{0}}\right)\right\rangle,
\end{aligned}
$$


where the first entry in $\boldsymbol{v}$ satisfies $\left|q_{0} 2^{\rho}\right|<2^{\gamma-\eta+\rho}$ and all the other entries satisfy $\left|x_{0} q_{0}\left(\frac{x_{i}}{x_{0}}-\frac{q_{i}}{q_{0}}\right)\right|=\left|x_{0} s_{i}\right| \approx 2^{\gamma+\rho-\eta}$.

However, the target solution is not necessarily the shortest nonzero vector in the lattice, and therefore is not necessarily discovered by lattice reduction. In particular, Minkowski tells us that $L$ has a nonzero vector of length at $\operatorname{most} \operatorname{det}(L)^{1 /(t+1)} \sqrt{t+1}<2^{(\rho+t \gamma) /(t+1)} \sqrt{t+1}=2^{\gamma+(\rho-\gamma) /(t+1)} \sqrt{t+1}$. This is shorter than our target solution when $t+1<\gamma / \eta$. In fact, heuristically, $L$ will tend to have exponentially (in $t$ ) many vectors of length $\operatorname{poly}(t) \operatorname{det}(L)^{1 /(t+1)}$, which obscure our target solution 5

On the other hand, when $t$ is large, $\boldsymbol{v}$ likely is the shortest vector in $L$, but known lattice reductions algorithms will not be able to find it efficiently. Specifically, as a rule of thumb, they require time roughly $2^{t / k}$ to output a $2^{k}$ approximation of the shortest vector. Since clearly there are exponentially (in $t$ ) many vectors in $L$ of length at most $\left\|x_{0}\right\| \sqrt{t+1}<2^{\gamma} \sqrt{t+1}$, which is about $2^{\eta-\rho}$ times longer than $\boldsymbol{v}$, we need better than a $2^{\eta-\rho}$ approximation. For $t \geq \gamma / \eta$, the time needed to guarantee a $2^{\eta}$ approximation (which is not even good enough to recover $\boldsymbol{v})$ is roughly $2^{\gamma / \eta^{2}}$. Thus setting $\gamma / \eta^{2}=\omega(\log \lambda)$ foils this attack.

Other known attacks are described in the full version. These attacks do not perform any better than the ones above, and our choice of parameters achieves at least $2^{\lambda}$ security against all of them.

\section{Making the Scheme Fully Homomorphic}

We follow Gentry's approach [6] for constructing a fully homomorphic encryption scheme from a somewhat homomorphic scheme $\mathcal{E}$ that is bootstrappable as per Definition 5. For reasons similar to those in Gentry's construction from 6], computing the decryption equation $m^{\prime} \leftarrow[c-\lfloor c / p\rceil]_{2}$ seems to require boolean circuits that are deeper (by a constant factor) than what our somewhat homomorphic scheme can handle. Hence we use Gentry's transformation to "squash the decryption circuit." In this transformation, we add to the public key some extra information about the secret key, and use this extra information to "post process" the ciphertext. The post-processed ciphertext can be decrypted more efficiently than the original ciphertext, thus making the scheme bootstrappable. We pay for this saving by having a larger ciphertext, and also by introducing another hardness assumption (basically assuming that the extra information in the public key does not help an attacker break the scheme).

\subsection{Squashing the Decryption Circuit}

Let $\kappa, \theta, \Theta$ be three more parameters, which are functions of $\lambda$. Concretely, below we use $\kappa=\gamma \eta / \rho^{\prime}, \theta=\lambda$, and $\Theta=\omega(\kappa \cdot \log \lambda) 6$ For a secret key $\mathrm{sk}^{*}=p$ and

\footnotetext{
${ }^{5}$ When $t$ is very small - e.g., $t=1$ - the information that one obtains from the two dimensional lattice is related to what one obtains from the continued fractions approach.

${ }^{6}$ When using the size-reduction optimization from Section 3.3 it is sufficient to use $\kappa=\gamma+2$, which would also make $\Theta$ smaller.
} 
public key $\mathrm{pk}^{*}$ from the original somewhat homomorphic scheme $\mathcal{E}^{*}$, we add to the public key a set $\boldsymbol{y}=\left\{y_{1}, \ldots, y_{\Theta}\right\}$ of rational numbers in $[0,2)$ with $\kappa$ bits of precision, such that there is a sparse subset $S \subset\{1, \ldots, \Theta\}$ of size $\theta$ with $\sum_{i \in S} y_{i} \approx 1 / p \quad(\bmod 2)$. We also replace the secret key by the indicator vector of the subset $S$. In more details, we modify the encryption scheme from Section 3 as follows:

KeyGen. Generate $\mathrm{sk}^{*}=p$ and $\mathrm{pk}^{*}$ as before. Set $x_{p} \leftarrow\left\lfloor 2^{\kappa} / p\right\rceil$, choose at random a $\Theta$-bit vector with Hamming weight $\theta, s=\left\langle s_{1}, \ldots, s_{\Theta}\right\rangle$, and let $S=\left\{i: s_{i}=1\right\}$.

Choose at random integers $u_{i} \in \mathbb{Z} \cap\left[0,2^{\kappa+1}\right), i=1, \ldots, \Theta$, subject to the condition that $\sum_{i \in S} u_{i}=x_{p}\left(\bmod 2^{\kappa+1}\right)$. Set $y_{i}=u_{i} / 2^{\kappa}$ and $\boldsymbol{y}=$ $\left\{y_{1}, \ldots, y_{\Theta}\right\}$. Hence each $y_{i}$ is a positive number smaller than two, with $\kappa$ bits of precision after the binary point. Also, $\left[\sum_{i \in S} y_{i}\right]_{2}=(1 / p)-\Delta_{p}$ for some $\left|\Delta_{p}\right|<2^{-\kappa}$.

Output the secret key sk $=s$ and public key $\mathrm{pk}=\left(\mathrm{pk}^{*}, \boldsymbol{y}\right)$.

Encrypt and Evaluate. Generate a ciphertext $c^{*}$ as before (i.e., an integer). Then for $i \in\{1, \ldots, \Theta\}$, set $z_{i} \leftarrow\left[c^{*} \cdot y_{i}\right]_{2}$, keeping only $n=\lceil\log \theta\rceil+3$ bits of precision after the binary point for each $z_{i}$. Output both $c^{*}$ and $\boldsymbol{z}=$ $\left\langle z_{1}, \ldots, z_{\Theta}\right\rangle$.

Decrypt. Output $m^{\prime} \leftarrow\left[c^{*}-\left\lfloor\sum_{i} s_{i} z_{i}\right\rceil\right]_{2}$.

Recall our definition of permitted polynomials from Section 3.2. We proved that our somewhat homomorphic scheme was correct for the $\operatorname{set} C\left(\mathcal{P}_{\mathcal{E}}\right)$ of circuit that compute permitted polynomials, and we now show that this is true also of the modified scheme.

Lemma 4. The modified scheme from above is correct for $C\left(\mathcal{P}_{\mathcal{E}}\right)$. Moreover, for every ciphertext $\left(c^{*}, \boldsymbol{z}\right)$ that is generated by evaluating a permitted polynomial, it holds that $\sum s_{i} z_{i}$ is within $1 / 4$ of an integer.

Proof. Fix public and secret keys, generated with respect to security parameter $\lambda$, with $\left\{y_{i}\right\}_{i=1}^{\Theta}$ the rational numbers in the public key and $\left\{s_{i}\right\}_{i=1}^{\Theta}$ the secretkey bits. Recall that the $y_{i}$ 's were chosen so that $\left[\sum_{i} s_{i} y_{i}\right]_{2}=(1 / p)-\Delta_{p}$ with $\left|\Delta_{p}\right| \leq 2^{-\kappa}$.

Fix a permitted polynomial $P\left(x_{1}, \ldots, x_{t}\right) \in \mathcal{P}_{\mathcal{E}}$, an arithmetic circuit $C$ that computes $P$, and $t$ ciphertexts $\left\{c_{i}\right\}_{i=1}^{t}$ that encrypt the inputs to $C$, and denote $c^{*}=$ Evaluate $\left(\mathrm{pk}, C, c_{1}, \ldots, c_{t}\right)$. We need to establish that

$$
\left\lfloor c^{*} / p\right\rceil=\left\lfloor\sum_{i} s_{i} z_{i}\right\rceil \quad(\bmod 2)
$$

where the $z_{i}$ 's are computed as $\left[c^{*} \cdot y_{i}\right]_{2}$ with only $\lceil\log \theta\rceil+3$ bits of precision after the binary point, so $\left[c^{*} \cdot y_{i}\right]_{2}=z_{i}-\Delta_{i}$ with $\left|\Delta_{i}\right| \leq 1 / 16 \theta$. We have 


$$
\begin{aligned}
{\left[\left(c^{*} / p\right)-\sum s_{i} z_{i}\right]_{2} } & =\left[\left(c^{*} / p\right)-\sum s_{i}\left[c^{*} \cdot y_{i}\right]_{2}+\sum s_{i} \Delta_{i}\right]_{2} \\
& =\left[\left(c^{*} / p\right)-c^{*} \cdot\left[\sum s_{i} y_{i}\right]_{2}+\sum s_{i} \Delta_{i}\right]_{2} \\
& =\left[\left(c^{*} / p\right)-c^{*} \cdot\left(1 / p-\Delta_{p}\right)+\sum s_{i} \Delta_{i}\right]_{2} \\
& =\left[c^{*} \cdot \Delta_{p}+\sum s_{i} \Delta_{i}\right]_{2}
\end{aligned}
$$

We claim that the final quantity inside the brackets has magnitude at most 1/8. By definition, since $c^{*}$ is a valid ciphertext output by a permitted polynomial, the value $c^{*} / p$ is within $1 / 8$ of an integer. Together, these facts imply the lemma.

To establish the claim, observe that $\left|\sum s_{i} \Delta_{i}\right| \leq \theta \cdot \frac{1}{16 \theta}=1 / 16$. Regarding $c^{*} \cdot \Delta_{p}$, recall that the output ciphertext $c^{*}$ is obtained by evaluating the polynomial $P$ on the input ciphertexts $c_{i}$ (as if $P$ was an integer polynomial). By the definition of a permitted polynomial, for any $\alpha \geq 1$, if $P$ 's inputs have magnitude at most $2^{\alpha\left(\rho^{\prime}+2\right)}$, its output has magnitude at most $2^{\alpha(\eta-4)}$ when its inputs have magnitude. In particular, when $P$ 's inputs are "fresh" ciphertexts, which have magnitude at most $2^{\gamma}, P^{\prime}$ 's output ciphertext $c^{*}$ has magnitude at most $2^{\gamma(\eta-4) /\left(\rho^{\prime}+2\right)}<2^{\kappa-4}$. Thus, $\left|c^{*} \cdot \Delta_{p}\right|<1 / 16$ and the claim follows.

\subsection{Bootstrapping Achieved!}

Theorem 3. Let $\mathcal{E}$ be the scheme above, and let $D_{\mathcal{E}}$ be the set of augmented (squashed) decryption circuits. Then, $D_{\mathcal{E}} \subset C\left(\mathcal{P}_{\mathcal{E}}\right)$.

In other words, $\mathcal{E}$ is bootstrappable. The proof is similar to Gentry's [5]6. By Theorem 1, we obtain homomorphic encryption schemes for circuits of any depth.

Proof. The goal is to express the modified decryption equation

$$
m^{\prime} \leftarrow c^{*}-\left\lfloor\sum s_{i} \cdot z_{i}\right\rceil \bmod 2
$$

as a permitted polynomial (i.e., one satisfying Equation (1)), and show that there is a polynomial-size circuit that computes this polynomial. Recall that $c^{*}$ is an integer, the $s_{i}$ 's are bits, and the $z_{i}$ 's are rational numbers in $[0,2)$, in binary representation with $n=\lceil\log \theta\rceil+3$ bits of precision after the binary point. Also, our parameter setting implies two promises - namely, that $\sum s_{i} \cdot z_{i}$ is within $1 / 4$ of an integer, and that only $\theta$ of the bits $s_{1}, \ldots, s_{\Theta}$ are nonzero.

We split the computation up into three steps:

1. For $i \in\{1, \ldots, \Theta\}$, set $a_{i} \leftarrow s_{i} \cdot z_{i}$ (i.e., $a_{i}=z_{i}$ when $s_{i}=1$ and $a_{i}=$ 0 otherwise). The $a_{i}$ 's are still rational numbers in $[0,2)$, given in binary representation with $n$ bits of precision after the binary point.

2. From the $\Theta$ rational numbers $\left\{a_{i}\right\}_{i=1}^{\Theta}$, generate other $n+1$ rational numbers $\left\{w_{j}\right\}_{j=0}^{n}$, each with less than $n$ bits of precision, such that $\sum_{j} w_{j}=\sum_{i} a_{i}$ $(\bmod 2)$.

3. Output $c^{*}-\left(\sum_{j} w_{j}\right) \bmod 2$. 
The first step can be performed with a 1-level sub-circuit of multiplication gates. However, the second and third steps require more complicated sub-circuits.

The problem of using a shallow boolean circuit to compute the sum $\sum_{i=1}^{k} r_{i}$ of $k$ rational numbers in binary representation is well-studied. A well-known technique uses the three-for-two trick (see [1] ), whereby a constant-depth circuit is used to transform three numbers of arbitrary bit-length into two numbers that are at most 1 bit longer, such that the sum of the two output numbers is the same as the sum of the three input numbers. (The output bits of the constant-depth circuit are linear or quadratic expressions with 3 monomials in the input bits.) By applying this trick at most $\left\lceil\log _{3 / 2} k\right\rceil+2$ times, one obtains two numbers $s_{1}$ and $s_{2}$ such that $s_{1}+s_{2}=\sum_{i=1}^{k} r_{i}$. Hence the total depth that it takes to reduce $k$ numbers to two numbers is $d^{\prime} \leq 2^{\left\lceil\log _{3 / 2} k\right\rceil+2}<8 k^{1 / \log (3 / 2)}<8 k^{1.71}$. The depth of the circuit needed to compute the final sum of two numbers is logarithmic in their bit-lengths, but if we are only interested in $\left\lfloor s_{1}+s_{2}\right\rfloor \bmod 2$ and have the promise that $s_{1}+s_{2}$ is within $1 / 4$ of an integer, this value can be computed by multivariate polynomial of degree 4 (and only nine terms). Overall, the circuit for computing $\left\lfloor\sum_{i=1}^{k} r_{i}\right\rceil \bmod 2$ corresponds to a polynomial of degree at most $d \leq 32 k^{1 / \log (3 / 2)}$. with coefficient vector having $l_{1}$-norm at most $27^{d}$. Unfortunately, this degree (with $k=\Theta$ ) is still too large for our scheme to handle. Hence we use Gentry's technique from [5] that takes advantage of the fact that all but $\theta$ of the $a_{i}$ 's are zero.

Denote the bit representation of each number $a_{i}$ by $a_{i, 0} a_{i,-1} a_{i,-2} \ldots a_{i,-n}$. That is, $a_{i}=\sum_{j=0}^{n} 2^{-j} a_{i,-j}$. The heart of this procedure is a subroutine for computing integers $W_{-j}, j=0,1, \ldots, n$, where $W_{-j}$ is the Hamming weight of the "column" of bits $\left(a_{1,-j}, a_{2,-j}, \ldots, a_{\Theta,-j}\right)$ (see an illustration in Figure 1). Since at most $\theta$ of the $a_{i}$ 's are nonzero, then the $W_{-j}$ 's are no larger than $\theta$, and hence can be represented by $\lceil\log (\theta+1)\rceil<n$ bits. By Lemma 5 below, every

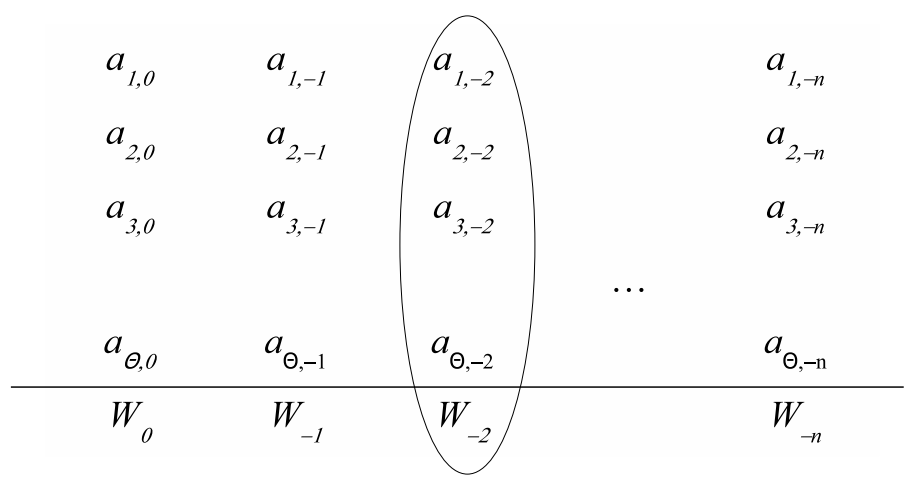

Fig. 1. The procedure for summing up the $a_{i}$ 's: The binary representation of the rational number $a_{i}$ is $a_{i, 0} a_{i,-1} a_{i,-2} \ldots a_{i,-n}$. The integer $W_{-j}$ is the Hamming weight of the column of bits $\left(a_{1,-j}, a_{2,-j}, \ldots, a_{\Theta,-j}\right)$. 
bit in the binary representation of $W_{-j}$ can be expressed as a polynomial of degree at most $\theta$ in the $\Theta$ variables $a_{i,-j}, i=1,2, \ldots, \Theta$. Moreover all of these polynomials can be computed simultaneously by an arithmetic circuit of size $O(\theta \cdot \Theta)$.

Once we have the $W_{-j}$ 's, the sum of the $a_{i}$ 's can be obtained by $\sum_{i} a_{i}=$ $\sum_{j} 2^{-j} W_{-j}$. For $j=0,1, \ldots, n$ we set $w_{j}=\left(2^{-j} \cdot W_{-j}\right) \bmod 2$, so the $w_{j}$ 's are rational numbers with $\lceil\log (\theta+1)\rceil<n$ bits of precision. We can now sum-up the $w_{j}$ 's using the three-for-two trick as above, this time with $k=n+1$, thus obtaining the sum of the $a_{i}$ 's mod 2 .

We conclude that the degree of the polynomials in the first step is two, the degree of polynomials in the second step is at most $\theta$, and the degree of the polynomial in the third step is at most

$$
32(n+1)^{1 / \log (3 / 2)}<32\lceil\log \theta+4\rceil^{1.71}<32 \log ^{2} \theta
$$

Therefore the total degree of the decryption circuit is bounded by $2 \cdot \theta \cdot 32 \log ^{2} \theta=$ $64 \theta \log ^{2} \theta$, and since we are using $\theta=\lambda$ we have degree at most $64 \lambda \log ^{2} \lambda$.

It follows that the augmented decryption circuits $D_{\mathcal{E}}$ (i.e., decryption followed by a single multiplication or addition, cf. Definition 4) can be expressed as polynomials of degree at most $128 \lambda \log ^{2} \lambda$ in the $\Theta$ variables $s_{i}$. Since the logarithm of $l_{1}$-norm of this polynomial is small in relation to $\eta$, and since $\Theta=\frac{\eta \gamma}{\rho} \cdot \omega(\log \lambda)<\lambda^{7}$ (and also $\tau<\lambda^{7}$ ) the argument in Remark 4 at the end of Section 3.2 (with $\alpha=128$ and $\beta=7$ ) indicates that we can get $D_{\mathcal{E}} \subset C\left(\mathcal{P}_{\mathcal{E}}\right)$, making the scheme bootstrappable, by setting $\eta=\rho \cdot \Theta\left(\lambda \log ^{2} \lambda\right)$.

It is left to show how to compute the $W_{j}$ 's using polynomials of degree no larger than $\theta$.

Lemma 5. Let $\boldsymbol{\sigma}=\left\langle\sigma_{1}, \sigma_{2}, \ldots, \sigma_{t}\right\rangle$ be a binary vector, let $W=W(\boldsymbol{\sigma})$ be the Hamming weight of $\boldsymbol{\sigma}$, and denote the binary representation of $W$ by $W_{n} \ldots W_{1} W_{0}$. (That is, $W=\sum_{i=0}^{n} 2^{i} W_{i}$ and all the $W_{i}$ 's are bits.)

Then for every $i \leq n$, the bit $W_{i}(\boldsymbol{\sigma})$ can be expressed as a binary polynomial of degree exactly $2^{i}$ in the variables $\sigma_{1}, \ldots, \sigma_{t}$. Moreover, there is an arithmetic circuit of size $2^{i} \cdot t$ that simultaneously computes all the polynomials for $W_{0}, \ldots, W_{i}$.

Proof. It is well known that the $i$ 'th bit in the binary representation of the Hamming weight of bit-vector $\boldsymbol{\sigma}$ is equal to $e_{2^{i}}(\boldsymbol{\sigma})$ modulo 2 , where $e_{k}(\cdot)$ is the $k$ 'th elementary symmetric polynomial, see Lemma 4 of [2]. That is,

$$
W_{i}(\boldsymbol{\sigma})=e_{2^{i}}(\boldsymbol{\sigma}) \bmod 2=\left(\sum_{|S|=2^{i}} \prod_{j \in S} \sigma_{j}\right) \bmod 2
$$

Clearly, the degree of $e_{2^{i}}$ is exactly $2^{i}$.

As for the "Moreover" part, we can compute the elementary symmetric polynomials in the $\sigma_{i}$ 's as the coefficients of the polynomial $P_{\boldsymbol{\sigma}}(z)=\prod_{i=1}^{t}\left(z-\sigma_{i}\right)$ in the auxiliary formal variable $z$, with $e_{k}(\boldsymbol{\sigma})$ being the coefficient of $z^{t-k}$. Conveniently, to compute only the first few bits $W_{0}, W_{1}, \ldots, W_{i}$, we can simply discard 
the lower-order terms in $P_{\boldsymbol{\sigma}}(z)$ - i.e., we do not need the coefficients of $z^{j}$ for $j<t-2^{i}$.

For example, one "dynamic programming" procedure for computing $W_{0}, W_{1}$, $\ldots, W_{i}$ (which can be trivially made into a circuit) would go as follows:

Input: bits $\sigma_{1}, \ldots, \sigma_{t}$

0. Initialization: Set $P_{0,0} \leftarrow 1$ and $P_{j, 0} \leftarrow 0$ for $j=1,2,3, \ldots, 2^{i}$ $/ / P_{j, k}$ is the $j$ 'th symmetric polynomial in $\sigma_{1} \ldots \sigma_{k}$

1. For $k=1,2, \ldots, t \quad / /$ incorporate $\sigma_{k}$

2. $\quad$ For $j=2^{i}$ down to 1 , set $P_{j, k} \leftarrow \sigma_{k} \times P_{j-1, k-1}+P_{j, k-1}$

3. Output $P_{1, t}, P_{2, t}, P_{4, t}, \ldots, P_{2^{i}, t}$

We can do a little better by using fast Fourier transform multiplication of polynomials. Using this technique, we can compute the entire polynomial $P_{\boldsymbol{\sigma}}(z)$ with complexity $t \cdot \operatorname{polylog}(t)$.

Remark 6. Note that our first circuit implementation of the procedure from above is not "shallow". Nonetheless, since it computes only "low degree polynomials" (i.e., up to degree $2^{i}$ ), then by Lemma 3 it is a permitted circuit.

\subsection{Security of the Squashed Scheme}

Putting the hint $\boldsymbol{y}$ in the public key induces another computational assumption, related to the sparse subset sum problem (SSSP) used by Gentry [5], and studied previously (sometimes under the name "low-weight" knapsack) in the context of server-aided cryptography [16] and in connection to the Chor-Rivest cryptosystem [18. We can easily avoid known attacks on the problem by choosing $\theta$ large enough to avoid brute-force attacks (and improvements using time-space trade-offs) and choosing $\Theta$ to be larger than $\omega(\log \lambda)$ times the bit-length of the rational numbers in the public key (which have length $\kappa$ ) 7

\section{Conclusion and Open Problems}

We described a fully homomorphic encryption scheme that uses only simple integer arithmetic. The primary open problem is to improve the efficiency of the scheme, to the extent that it is possible while preserving the hardness of the approximate-gcd problem.

\section{References}

1. Alexi, W., Chor, B., Goldreich, O., Schnorr, C.-P.: Rsa and rabin functions: Certain parts are as hard as the whole. SIAM J. Comput. 17(2), 194-209 (1988)

2. Boyar, J., Peralta, R., Pochuev, D.: On the multiplicative complexity of boolean functions over the basis $(\wedge, \oplus, 1)$. Theor. Comput. Sci. 235(1), 43-57 (2000)

\footnotetext{
${ }^{7}$ Note that the SSSP instance and the approximate-GCD instance share the same integer $p$, but this is not a problem since SSSP is considered hard even if the attacker knows $p$.
} 
3. Cachin, C., Micali, S., Stadler, M.: Computationally private information retrieval with polylogarithmic communication. In: Stern, J. (ed.) EUROCRYPT 1999. LNCS, vol. 1592, pp. 402-414. Springer, Heidelberg (1999)

4. Coppersmith, D.: Small solutions to polynomial equations, and low exponent RSA vulnerabilities. J. Cryptology 10(4), 233-260 (1997)

5. Gentry, C.: A fully homomorphic encryption scheme. PhD thesis, Stanford University (2009), http://crypto.stanford.edu/craig

6. Gentry, C.: Fully homomorphic encryption using ideal lattices. In: STOC 2009, pp. 169-178. ACM, New York (2009)

7. Goldwasser, S., Micali, S.: Probabilistic encryption. Journal of Computer and System Sciences 28(2), 270-299 (1984)

8. Håstad, J., Impagliazzo, R., Levin, L.A., Luby, M.: A pseudorandom generator from any one-way function. SIAM J. Comput. 28(4), 1364-1396 (1999)

9. Howgrave-Graham, N.: Approximate integer common divisors. In: Silverman, J.H. (ed.) CaLC 2001. LNCS, vol. 2146, pp. 51-66. Springer, Heidelberg (2001)

10. Ishai, Y., Paskin, A.: Evaluating branching programs on encrypted data. In: Vadhan, S.P. (ed.) TCC 2007. LNCS, vol. 4392, pp. 575-594. Springer, Heidelberg (2007)

11. Karp, R.M., Ramachandran, V.: A Survey of Parallel Algorithms for SharedMemory Machines. Technical Report CSD-88-408, UC Berkeley (1988)

12. Knuth, D.E.: Seminumerical Algorithms, 3rd edn. The Art of Computer Programming, vol. 2. Addison-Wesley, Reading (1997)

13. Lagarias, J.C.: The computational complexity of simultaneous diophantine approximation problems. SIAM J. Comput. 14(1), 196-209 (1985)

14. Lenstra, A.K.: Factoring multivariate polynomials over algebraic number fields. SIAM J. Comput. 16(3), 591-598 (1987)

15. Lindell, Y., Pinkas, B.: A proof of security of yao's protocol for two-party computation. J. Cryptology 22(2) (2009)

16. Nguyen, P.Q., Shparlinski, I.: On the insecurity of a server-aided RSA protocol. In: Boyd, C. (ed.) ASIACRYPT 2001. LNCS, vol. 2248, pp. 21-35. Springer, Heidelberg (2001)

17. Nguyen, P.Q., Stern, J.: The two faces of lattices in cryptology. In: Silverman, J.H. (ed.) CaLC 2001. LNCS, vol. 2146, pp. 146-180. Springer, Heidelberg (2001)

18. Nguyen, P.Q., Stern, J.: Adapting density attacks to low-weight knapsacks. In: Roy, B. (ed.) ASIACRYPT 2005. LNCS, vol. 3788, pp. 41-58. Springer, Heidelberg (2005)

19. Regev, O.: New lattice-based cryptographic constructions. JACM 51(6), 899-942 (2004)

20. Rivest, R., Adleman, L., Dertouzos, M.: On data banks and privacy homomorphisms. In: Foundations of Secure Computation, pp. 169-177. Academic Press, London (1978)

21. van Dijk, M., Gentry, C., Halevi, S., Vaikuntanathan, V.: Fully homomorphic encryption over the integers. Cryptology ePrint Archive, Report 2009/616 (2009), http://eprint.iacr.org/2009/616

22. Yao, A.C.: Protocols for secure computations. In: 23rd Annual Symposium on Foundations of Computer Science - FOCS 1982, pp. 160-164. IEEE, Los Alamitos (1982) 